\title{
REMARKS ON RINGS OF CONSTANTS OF DERIVATIONS
}

\author{
WEI LI
}

(Communicated by Louis J. Ratliff, Jr.)

\begin{abstract}
Let $k$ be a field of characteristic $p>0$ and $D \neq 0$ a family of $k$-derivations of $k[x, y]$. We prove that $k[x, y]^{D}$, the ring of constants with respect to $D$, is a free $k\left[x^{p}, y^{p}\right]$-module of rank $p$ or 1 and $k[x, y]^{D}=$ $k\left[x^{p}, y^{p}, f_{1}, \ldots, f_{p-1}\right]$ for some $f_{1}, \ldots, f_{p-1} \in k[x, y]^{D}$.
\end{abstract}

In this note we generalize some results of $A$. Nowicki and $M$. Nagata on rings of constants for $k$-derivations of $k[x, y]$ in their paper [N-N]. Before stating their results let us set up notations which will be used throughout this paper.

We assume that $k$ is always a field of characteristic $p>0$. A $k$-linear map $d: k\left[x_{1}, \ldots, x_{n}\right] \rightarrow k\left[x_{1}, \ldots, x_{n}\right]$ is called a $k$-derivation of $k\left[x_{1}, \ldots, x_{n}\right]$ if $d$ satisfies the condition:

$$
d(f g)=d(f) g+f d(g), \quad \forall f, g \in k\left[x_{1}, \ldots, x_{n}\right] .
$$

If $D$ is a family of $k$-derivations of $k\left[x_{1}, \ldots, x_{n}\right]$, we denote by $k\left[x_{1}, \ldots, x_{n}\right]^{D}$ the set of constants of $D$ in $k\left[x_{1}, \ldots, x_{n}\right]$, i.e.,

$$
k\left[x_{1}, \ldots, x_{n}\right]^{D}=\left\{f \in k\left[x_{1}, \ldots, x_{n}\right] \mid d(f)=0, \forall d \in D\right\} .
$$

If $D=\{d\}$, we simply write $k\left[x_{1}, \ldots, x_{n}\right]^{D}=k\left[x_{1}, \ldots, x_{n}\right]^{d}$.

It is easy to see that for any family $D$ of $k$-derivations of $k\left[x_{1}, \ldots, x_{n}\right]$, $k\left[x_{1}^{p}, \ldots, x_{n}^{p}\right] \subseteq k\left[x_{1}, \ldots, x_{n}\right]^{D}$; and $k\left[x_{1}, \ldots, x_{n}\right]^{D}$, as a $k\left[x_{1}^{p}, \ldots, x_{n}^{p}\right]-$ submodule of $k\left[x_{1}, \ldots, x_{n}\right]$, is a finitely generated $k\left[x_{1}^{p}, \ldots, x_{n}^{p}\right]$-module. It is interesting to consider relations between these two rings. Among other things, in their recent paper [N-N] Nowicki and Nagata prove the following results:

A. [N-N, Proposition 4.2] If $D \neq 0, p=2$, then there exists an $f \in k[x, y]$ such that $k[x, y]^{D}=k\left[x^{p}, y^{p}, f\right]$;

B. [N-N, Theorem 4.4] $k[x, y]^{d}$ is a free $k\left[x^{p}, y^{p}\right]$-module for any single $k$-derivation $d$.

In this note we generalize these two results; namely, we prove the following theorem.

Received by the editors November 21, 1988.

1980 Mathematics Subject Classification (1985 Revision). Primary 13B10.

Key words and phrases. Derivations, rings of constants of derivations. 
Theorem. Let $k$ be a field of characteristic $p>0, D \neq 0$ a family of $k$ derivations of $k[x, y]$. Then

(i) $k[x, y]^{D}$ is a free $k\left[x^{p}, y^{p}\right]$-module of rank $p$ or 1 ;

(ii) there exist $g_{1}, \ldots, g_{p-1} \in k[x, y]^{D}$ such that $k[x, y]^{D}=k\left[x^{p}, y^{p}, g_{1}\right.$, $\left.\ldots, g_{p-1}\right]$.

Note that $k[x, y]^{D}$ is always a two-dimensional normal domain; hence it is Cohen-Macaulay. It is a natural question whether $k[x, y]^{D}$ is regular. We shall give a negative answer to this question by giving an example which is not locally UFD. We shall also give an example showing that for $n \geq 3, k\left[x_{1}, \ldots, x_{n}\right]^{D}$ may not be Cohen-Macaulay.

To prove the theorem, we need the following theorem (see [M], p. 140).

Theorem A. Let $A$ be a regular local ring, and $B$ a domain containing $A$ which is a finite A-module. Then $B$ is free over $A$ iff $B$ is Cohen-Macaulay.

Proof of theorem. Denote $A=k[x, y]^{D} . A$ is a finitely generated $k\left[x^{p}, y^{p}\right]-$ module, as being a $k\left[x^{p}, y^{p}\right]$-submodule of $k[x, y]$. Let $\mathscr{M}$ be a maximal ideal of $k\left[x^{p}, y^{p}\right]$. Then $k\left[x^{p}, y^{p}\right]_{\mathscr{M}}$ is a regular local ring, $A_{\mathscr{M}}$ is a finite $k\left[x^{p}, y^{p}\right]_{\mathscr{M}}$-module. Since $A$ is a normal domain, $A_{\mathscr{M}}$ is normal. Note that $\operatorname{dim} A=2$. Hence, by [M, Theorem 38, p. 124], $A$ is Cohen-Macaulay. Therefore, by using Theorem A, $A_{\mathscr{M}}$ is a free $k\left[x^{p}, y^{\underline{D}}\right]_{\mathscr{M}}$-module. It follows that $A$ is projective over $k\left[x^{p}, y^{p}\right]$. Thus, by Seshadri [S] (see [L]), $A=$ $k[x, y]^{D}$ is a free $k\left[x^{p}, y^{p}\right]$-module.

Assume that $k[x, y]^{D}$, as a free $k\left[x^{p}, y^{p}\right]$-module, has rank $s$. Let $f_{1}, \ldots$, $f_{s} \in k[x, y]^{D}$ be a set of generators of $k\left[x^{p}, y^{p}\right]$-module $k[x, y]^{D}$. Denote $F=k\left(x^{p}, y^{p}\right)\left[f_{1}, \ldots, f_{s}\right]$. Then $F$ is the field of quotients of $k[x, y]^{D}$ since $f_{1}, \ldots, f_{s}$ are integral over $k\left(x^{p}, y^{p}\right)$. Denote $t=\left[F: k\left(x^{p}, y^{p}\right)\right]$. Then $t=1$, $p$, or $p^{2}$. But $t=p^{2}$ is impossible, as $D \neq 0$. Note that

$$
F=k\left(x^{p}, y^{p}\right) \otimes_{k\left[x^{p}, y^{p}\right]} k[x, y]^{D}
$$

and that $k[x, y]^{D}$ is a free $k\left[x^{p}, y^{p}\right]$-module. If follows that $f_{1}, \ldots, f_{s}$ is a basis of $F$ over $k\left(x^{p}, y^{p}\right)$, and hence $s=t$. Therefore $s=1$ or $p$, proving (i).

To prove (ii), we may assume that $s=p$ and $k[x, y]^{D}$ is generated by $f_{1}, \ldots, f_{p}$ as a $k\left[x^{p}, y^{p}\right]$-module. We have

$$
1=\sum_{i=1}^{p} r_{i} f_{i}, \quad r_{i} \in k\left[x^{p}, y^{p}\right], \quad i=1, \ldots, p .
$$

This means that $\left(r_{1}, \ldots, r_{p}\right)$ is a unimodular row over $k[x, y]$. Since $k[x, y]$ is integral over $k\left[x^{p}, y^{p}\right],\left(r_{1}, \ldots, r_{p}\right)$ must be a unimodular row over $k\left[x^{p}, y^{p}\right]$. Using Seshadri [S] (see [L]) again, $\left(r_{1}, \ldots, r_{p}\right)$ can be completed as a $p \times p$ matrix over $k\left[x^{p}, y^{p}\right]$ with determinant in $k^{*}$. Then a basis change 
gives a generating set $\left\{g_{1}=1, g_{2}, \ldots, g_{p}\right\}$ of $k[x, y]^{D}$ as a $k\left[x^{p}, y^{p}\right]$-module. Hence $k[x, y]^{D}=k\left[x^{p}, y^{p}, g_{2}, \ldots, g_{p}\right]$.

Note that for any family $D$ of $k$-derivations, $k[x, y]^{D}$ is a two-dimensional normal domain; hence it is Cohen-Macaulay. We here give an example showing that $k[x, y]^{D}$ may not be regular. Note that if $k$ is of characteristic zero, $k[x, y]^{D}$ is always regular (see $\left.[\mathrm{N}-\mathrm{N}]\right)$.

Example 1. [N-N, Example 4.3] Let $k$ be a field of characteristic $p>0$ and let $d$ be the $k$-derivation of $k[x, y]$ defined by

$$
d(f)=\frac{\partial f}{\partial x} x+\frac{\partial f}{\partial y} y, \quad f \in k[x, y] .
$$

We are going to show that $k[x, y]^{d}$ is not locally UFD. This implies that $k[x, y]^{d}$ is not regular. Let $\mathscr{M}=(x, y) k[x, y] \cap k[x, y]^{d}$. Since $k[x, y]$ is integral over $k[x, y]^{d}, \mathscr{M}$ is a maximal ideal of $k[x, y]^{d}$. Denote $A=k[x, y]^{d}$. We claim that $A_{\mathscr{M}}$ is not a UFD. It is easy to check that $x^{p}, y^{p}, x^{p-1} y$, $x y^{p-1} \in A$. They are all irreducible elements in $A$ since any proper factor of them is not in $k[x, y]^{d}=A$. We want to prove that $x^{p}, y^{p}, x^{p-1} y$, and $x y^{p-1}$ are all irreducible in $A_{\mathscr{M}}$. Suppose, for example, that $x^{p-1} y$ is reducible in $A_{\mathscr{M}}$. Then

where $g_{1}, g_{2} \in \mathscr{M}, h_{1}, h_{2} \in A-\mathscr{M}$. Hence

$$
x^{p-1} y=\frac{g_{1}}{h_{1}} \cdot \frac{g_{2}}{h_{2}}
$$

$$
g_{1} g_{2}=x^{p-1} y h_{1} h_{2} \text {. }
$$

We may assume that $y \mid g_{2}$ (in $k[x, y]$ ). Then $g_{1}=x^{r} h$, where $1 \leq r \leq p-1$ and $h \in k[x, y]$ such that $h \mid h_{1} h_{2}$ (in $\left.k[x, y]\right)$. Since $h_{1}, h_{2} \in A-\mathscr{M}, h_{1} h_{2} \notin$ $(x, y) k[x, y]$, hence $h \notin(x, y) k[x, y]$. We have $d\left(x^{r} h\right)=d\left(g_{1}\right)=0$ since $g_{1} \in A$. But

$$
\begin{aligned}
d\left(x^{r} h\right) & =\frac{\partial}{\partial x}\left(x^{r} h\right) x+\frac{\partial}{\partial y}\left(x^{r} h\right) y \\
& =r x^{r} h+x^{r+1} \frac{\partial h}{\partial x}+x^{r} y \frac{\partial h}{\partial y} .
\end{aligned}
$$

Therefore

$$
r h=-x \frac{\partial h}{\partial x}-y \frac{\partial h}{\partial y} \in(x, y) k[x, y] .
$$

Noticing that $r \not \equiv 0(p)$, we have $h \in(x, y) k[x, y]$, which is a contradiction. Hence $x^{p-1} y$ is irreducible in $A_{\mathscr{M}}$. Similarly, $x^{p}, y^{p}$, and $x y^{p-1}$ are all irreducible in $A_{\mathscr{K}}$. We have

$$
(x y)^{p}=x^{p} \cdot y^{p}=\left(x^{p-1} y\right) \cdot\left(x y^{p-1}\right) .
$$

To prove that $A_{\mathscr{M}}$ is not UFD, it suffices to prove that neither $x^{p} / x^{p-1} y$ nor $x^{p} / x y^{p-1}$ is a unit in $A_{\mathscr{M}}$. But $x^{p} / x^{p-1} y=x / y, x^{p} / x y^{p-1}=x^{p-1} / y^{p-1}$. 
Therefore, to prove that $A_{\mathscr{M}}$ is not UFD, it suffices to prove that $x^{p-1} / y^{p-1} \notin$ $A_{\mathscr{M}}$. Suppose $x^{p-1} / y^{p-1} \in A_{\mathscr{M}}$. Then there exists an $f \in k[x, y]$ such that $x^{p-1} f \in A, y^{p-1} f \in A-\mathscr{M}$. But $y^{p-1} f \in(x, y) k[x, y]$, implying that $y^{p-1} f \in(x, y) k[x, y] \cap A=\mathscr{M}$. This is a contradiction, proving the claim that $A_{\mathscr{M}}$ is not UFD.

If $n \geq 3, k\left[x_{1}, \ldots, x_{n}\right]^{D}$ may not be Cohen-Macaulay, as will be shown by the following example:

Example 2 [N-N, Example 4.6]. Let $n \geq 3$, and let $d$ be the $k$-derivation of $k\left[x_{1}, \ldots, x_{n}\right]$ defined by

$$
d(f)=\sum_{i=1}^{n} \frac{\partial f}{\partial x_{i}} x_{i}^{p}, \quad f \in k\left[x_{1}, \ldots, x_{n}\right] .
$$

Denote $A=k\left[x_{1}^{p}, \ldots, x_{n}^{p}\right], B=k\left[x_{1}, \ldots, x_{n}\right]^{d}$. It is proved in [N-N $]$ that $B$ is not a free $A$-module. There exists a maximal ideal $\mathscr{M}$ of $A$ such that $B_{\mathscr{M}}$ is not a free $A_{\mathscr{M}}$-module. Using Theorem A, $B_{\mathscr{M}}$ is not Cohen-Macaulay. Hence there exists a maximal ideal $\mathscr{N} \subset B$ such that $\mathscr{N} \cap(A-\mathscr{M})=\varnothing,\left(B_{\mathscr{M}}\right)_{\mathscr{N}}=B_{\mathscr{N}}$ is not Cohen-Macaulay. Therefore $B$ is not Cohen-Macaulay.

Indeed one can prove, using Theorem $\mathrm{A}$, that for any $D, k\left[x_{1}, \ldots, x_{n}\right]^{D}$ is Cohen-Macaulay if and only if it is free over $k\left[x_{1}^{p}, \ldots, x_{n}^{p}\right]$.

\section{REFERENCES}

[L] T. Y. Lam, Serre's conjecture, Lecture Notes in Math., no. 635, Springer-Verlag, New York, 1978.

[M] H. Matsumura, Commutative algebra, 2nd edition, Benjamin Cummings, 1980.

[N-N] A. Nowicki and M. Nagata, Rings of constants for $k$-derivations in $k\left[x_{1}, \ldots, x_{n}\right], \mathrm{J}$. Math. Kyoto Univ. 28 (1988), 111-118.

[S] C. S. Seshadri, Triviality of vector bundles over the affine space $K^{2}$, Proc. Nat. Acad. Sci. U.S.A. 44 (1958), 456-458.

Department of Mathematics, Purdue University, West Lafayette, Indiana 47907 